\title{
Femto-Photography: Capturing Light in Motion
}

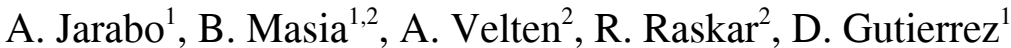 \\ ${ }^{I}$ Graphics and Imaging Lab-Advanced Computer Graphics Group (GIGA) \\ Instituto de Investigación en Ingeniería de Aragón (I3A) \\ Universidad de Zaragoza, Mariano Esquillor s/n, 50018, Zaragoza, Spain. \\ Tel. +34-976762707, Fax +34-976762043, e-mail: ajarabo@unizar.es \\ ${ }^{2}$ MIT Media Lab \\ Massachusetts Institute of Technology (MIT). \\ Cambridge MA, USA
}

\begin{abstract}
We show a technique to capture ultrafast movies of light in motion and synthesize physically valid visualizations. The effective exposure time for each frame is under two picoseconds (ps). Capturing a 2D video with this time resolution is highly challenging, given the extermely low SNR associated with a picosecond exposure time, as well as the absence of 2D cameras that can provide such a shutter speed. We re-purpose modern imaging hardware to record an ensemble average of repeatable events that are synchronized to a streak tube, and we introduce reconstruction methods to visualize propagation of light pulses through macroscopic scenes. Capturing two-dimensional movies with picosecond resolution, we observe many interesting and complex light transport effects, including multibounce scattering, delayed mirror reflections, and subsurface scattering. We notice that the time instances recorded by the camera, i.e. "camera time" is different from the the time of the events as they happen locally at the scene location, i.e. world time. We introduce a notion of time warp between the two space time coordinate systems, and rewarp the space-time movie for a different perspective.
\end{abstract}

